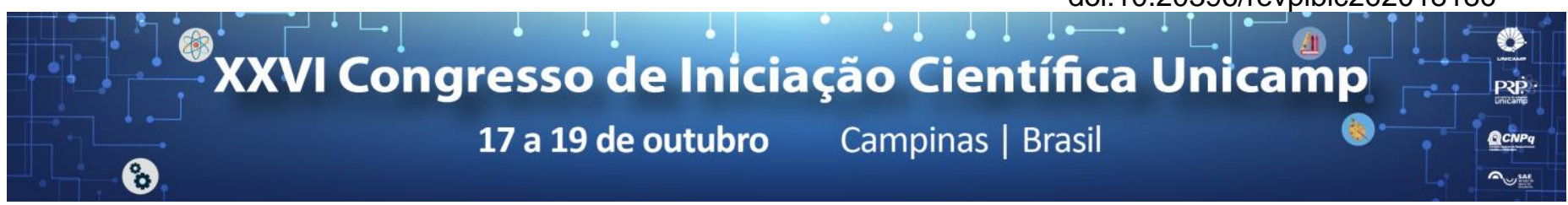

\title{
Status and challenges for the molecular characterization of Fannia (Insecta, Diptera, Fanniidae) in Brazil
}

\section{Bárbara M. C. Maia*, Taís Madeira-Ott, Patrícia J. Thyssen}

\begin{abstract}
Both the advancement of technology associated with molecular characterization studies of species have contributed to the increased use of the Barcode DNA method for identifying individuals. For some areas, such as forensics, species determination is essential for information regarding the time of death. In this study, Fannia nucleotide sequences available in public databases were quantified and used to evaluate the scenario and the challenge to establish molecular research with fanids in Brazil.
\end{abstract}

Key words:

DNA barcode, taxonomic impediment, forensic entomology.

\section{Introduction}

Fannia Robineau-Desvoidy is the most diverse genus of the Fanniidae family (Insecta, Diptera, Muscomorpha), representing about $90 \%$ of the species richness of Neotropical fanids ${ }^{1}$. Although it can develop in different substrates, the great majority of species present saprophagous habits ${ }^{2}$. Since they are seen rearing in cadavers, they may have relevance in the forensic field, especially when providing information to estimate the postmortem interval2,3. Due to the difficulty of collecting and rearing specimens in the laboratory, there is little information available about fanids and, consequently, on their taxonomy, thus making more difficult the species identification by forensics experts and other professionals.

The molecular characterization of species of forensic importance using the Barcode DNA method has become a very useful and effective identification practice that can offer a specific reliable diagnosis ${ }^{4}$. But for this there is a need for a nucleotide sequence database that provides information on most of the diversity of target insects for identification.

The objective of this study was to determine how many and which species of Fannia registered for our country and classified as of forensic importance have nucleotide sequences deposited in the Genbank database. In addition and through this analysis, the current status of Brazilian research on the molecular characterization of fanids was evaluated.

\section{Results and Discussion}

First, a search in digital databases (including Pubmed, Google Scholar, JStor) was performed using the following descriptors, isolated or combined: Fannia, necrophagous, identification, forensic, description, development, postmortem interval, barcode, Brazil, Neotropical. The research returned 26 species of Fannia classified as of forensic importance to Brazil.

Subsequently, the GenBank database was consulted and 71 nucleotide sequences of the mitochondrial cytochrome oxidase I (COI) gene (= barcode) were found for Fannia. None of the sequences originate from Brazilian studies and all belong to only three species of Fannia, whose distribution to Brazil has already been recorded: F. pusio, F. canicularis and F. scalaris. Our analysis show the formation of three clades (Fig. 1) quite consistent with the operational taxonomic units investigated here. Additionally, genetic distance analyzes showed intervals between 0 and $1 \%$ and 9.5 and $15 \%$ intra and interspecific, respectively, demonstrating that the molecular characterization for Fannia species can be promising.

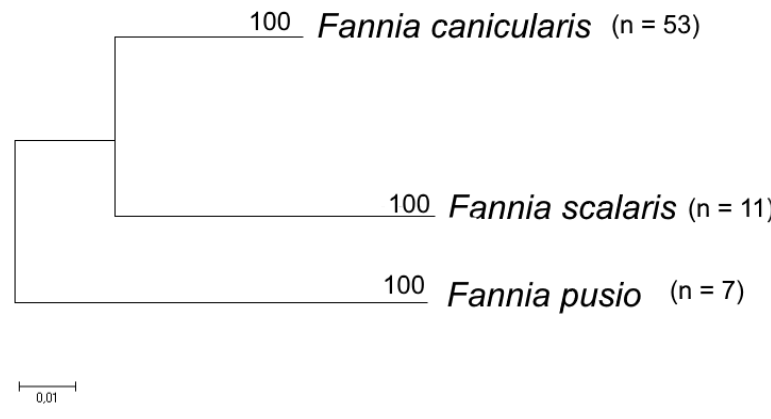

Figure 1. Neighbor-joining tree inferred for a set of $\mathrm{CO}$ (barcode) sequences of the species F. pusio, F. canicularis and F. scalaris deposited at Genbank.

\section{Conclusions}

This study shows that there is no representation of Fannia sequences found in Brazilian territory in the molecular databases. Possibly, this reality is a reflection of the little incentive to the formation of taxonomists for this group. Several species are found associated with corpses but are not used in criminal investigations for lack of alternatives for their identification. The inclusion and availability of a larger number of species sequences in the databases could contribute to minimize the taxonomic impediment of these dipterans in Brazil.

\footnotetext{
${ }^{1}$ Grisales, D; Lecheta, M. C.; Aballay, F. H. and Carvalho, C. J. B. Zoologia 2016, 33. e20160054.

${ }^{2}$ Alves, A. C.; Santos, W. E. and Creão-Duarte, A. J. Entomotropica. 2014, 29 77-94.

${ }^{3}$ Vasconcelos, S. D.; Soares, T. F. and Costa, D. L. Int. J. Leg. Med. 2014, 128 , 229-233.

${ }^{4}$ Hebert, P. D. N.; Cywinska, A.; Ball, S. L. and Dewards, J. R. Proc. Royal Soc. London. 2003, 270, 313-321.
} 